\title{
Corrigendum: Iron Acquisition Mechanisms and Their Role in the Virulence of Burkholderia Species
}

\author{
Aaron T. Butt and Mark S. Thomas* \\ Department of Infection, Immunity and Cardiovascular Disease, Faculty of Medicine, Dentistry and Health, University of \\ Sheffield, Sheffield, United Kingdom
}

Keywords: Burkholderia, iron, siderophores, haem uptake, cystic fibrosis, melioidosis

\section{A Corrigendum on}

Iron Acquisition Mechanisms and Their Role in the Virulence of Burkholderia Species by Butt, A. T., and Thomas, M. S. (2017). Front. Cell. Infect. Microbiol. 7:460. doi: $10.3389 /$ fcimb.2017.00460

In the original article, there was a mistake in the legend for Figure 2 as published. Ornibactins and malleobactins contain two hydroxamates groups, not one, and a single $\alpha$-hydroxycarboxylate group, not two $\beta$-hydroxycarboxylate groups. The correct legend appears below. The authors apologize for this error and state that this does not change the scientific conclusions of the article in any way.

Figure 2: Structure of siderophores produced by Burkholderia species. (A) Ornibactins contain an N-terminal ornithine that is acylated with a C4, C6, or C8 $\beta$-hydroxycarboxylic acid on the $\delta$-amino nitrogen atom, giving rise to ornibactin- $44,-\mathrm{C} 6$, or $-\mathrm{C} 8$. The $\delta$-amino nitrogen atom is also hydroxylated. The other three amino acids in the tetrapeptide are D-hydroxyaspartate, $\mathrm{L}$-serine, and the C-terminal ornithine that is formylated and hydroxylated on the $\delta$-amino nitrogen atom and the carboxyl group is conjugated to putrescine. As with the malleobactins, they contain two bidentate hydroxamate ligands and a single bidentate $\alpha$-hydroxycarboxylate ligand. (B) Malleobactin E, the siderophore-active malleobactin congener of $B$. thailandensis. (C) The siderophore-active malleobactin congener of B. xenovorans, tentatively referred to here as "malleobactin X." (D) Cepaciachelin contains two 2,3-DHBA groups that form amide linkages with the two amino groups of lysine, which in turn is conjugated to a molecule of putrescine (1,4-diaminobutane) on its $\alpha$-carboxyl group. (E) Pyochelin contains two less commonly occurring bidentate iron-chelating groups (2-hydroxyphenyl thiazoline and N-methylthiazolidine4-carboxylate). (F) Cepabactin, a cyclic hydroxamate bidentate siderophore. Chemical groups that distinguish the ornibactins and malleobactins are indicated in red circles or ellipses.

The original article has been updated. Accepted: 08 August 2018

Published: 24 August 2018

Citation:

Butt AT and Thomas MS (2018)

Corrigendum: Iron Acquisition Mechanisms and Their Role in the Virulence of Burkholderia Species. Front. Cell. Infect. Microbiol. 8:305. doi: 10.3389/fcimb.2018.00305

Conflict of Interest Statement: The authors declare that the research was conducted in the absence of any commercial or financial relationships that could be construed as a potential conflict of interest.

Copyright $\odot 2018$ Butt and Thomas. This is an open-access article distributed under the terms of the Creative Commons Attribution License (CC BY). The use, distribution or reproduction in other forums is permitted, provided the original author(s) and the copyright owner(s) are credited and that the original publication in this journal is cited, in accordance with accepted academic practice. No use, distribution or reproduction is permitted which does not comply with these terms. 